\title{
Histopathology of Chlamydia trachomatis salpingitis after primary and repeated reinfections in the monkey subcutaneous pocket model
}

\author{
D. L. Patton and C.-C. Kuo* \\ Departments of Obstetrics and Gynecology and ${ }^{*}$ Pathobiology, University of Washington, Seattle, \\ Washington 98195, USA
}

\begin{abstract}
Summary. Monkeys with subcutaneously autotransplanted salpingeal fimbrial tissues were subjected to primary and repeated infections with Chlamydia trachomatis. The inflammatory response after primary inoculation was characterized by infiltration with polymorphonuclear leucocytes in the acute phase and mononuclear cells in the chronic phase. However, the inflammatory response after repeated infections was dominated by a mononuclear cell infiltration with a conspicuous absence of the initial phase of polymorphonuclear leucocyte infiltration. The remarkable findings of repeated infections were plasma cell infiltration, lymphoid follicle formation, and increased fibroblast activity resulting in extensive fibrosis. These findings are similar to those described for monkeys inoculated directly into the oviducts with $C$. trachomatis and support our original hypothesis that, after chlamydial infection, the tissue damage is provoked by immune-mediated mechanisms.
\end{abstract}

Keywords: monkey; Chlamydia; salpingitis; histopathology; immunopathology

\section{Introduction}

We have previously described a subcutaneous pocket model of oviducal implants in rhesus and pigtailed monkeys for studying Chlamydia trachomatis salpingitis (Patton et al., 1987a). Briefly, in this model the fimbrial tissues of the salpinx were autotransplanted to the subcutis region of the abdominal skin. The implants became vascularized and were walled off within a cyst into which $C$. trachomatis was inoculated directly. The transplanted salpingeal epithelium has been shown to be susceptible to the infection with $C$. trachomatis as indicated by reisolation of the organism. The inoculated animals have developed both serum and local (in the pocket fluid) antibody responses that are specific to the infecting strain.

Descriptions of the histopathology of chlamydial salpingitis in man are poorly documented. To characterize the natural course of primary and repeated reinfections regarding the histopathology and immunopathology of the Fallopian tube to chlamydial infection, we have utilized the subcutaneous pocket model to study the pathogenesis of chronic salpingitis and the resulting scarring that leads to tubal infertility.

In this report, we present the histopathological findings of the subcutaneous fimbrial transplants following primary and repeated infections with $C$. trachomatis.

\section{Materials and Methods}

The subcutaneous fimbrial transplant monkey model. The procedure of implanting the oviduct fimbria in the subcutis of the anterior abdominal skin of monkeys has been described (Patton et al., 1987a). Rhesus (Macaca mulatta) 
and pig-tailed (Macaca nemestrina) monkeys were used in this study. As many as 20 autografts could be created in any one monkey by using both fimbriae.

Inoculation of fimbrial transplants. The monkeys were anaesthetized with ketamine/xylazine and the inoculum containing 10,000 inclusion forming units of serovar $\mathrm{E}(\mathrm{UW}-5 / \mathrm{Cx})$ strain of $C$. trachomatis was injected directly through the skin into the fimbrial pockets. Control pockets were inoculated with $\mathrm{HeLa}$ cell materials because the organisms were grown in HeLa cell culture or u.v.-inactivated organisms. Some pockets were inoculated with the same organism for a 2 nd and 3 rd time at 30-day intervals following primary challenge (homologous challenge).

Collection of specimens. Individual pockets were surgically excised at various times after inoculation as indicated below. Since no single monkey could contribute enough pockets for the all intervals studied, several monkeys (11 rhesus and 1 pig-tailed) were used to cover the entire study period. Because no significant differences had been found between the two species of monkeys studied (Patton et al., 1987a), the experimental results have been combined. Two or 3 pockets were studied at each time interval. Each transplant was used for either isolation or histopathology. Tissues for isolation were placed in Chlamydia transport medium (sucrose-phosphate-glutamate) and those for histopathology in Carnoy's fixative. Venous blood was collected weekly throughout the entire study period of 12-16 weeks. The serum was stored at $-20^{\circ} \mathrm{C}$ and used for serology studies.

Isolation and serology. The biopsy tissue was homogenized in $0.7 \mathrm{ml}$ Chlamydia transport medium using a powerdriven miniature tissue grinder (Con-Torque, Eberbach Corp., Ann Arbor, MI, USA). The coarse tissue debris was removed by low speed centrifugation ( $150 \mathrm{~g}$ for $8 \mathrm{~min}$ ). The supernatant was used for inoculation of HeLa 229 cell culture for isolation of $C$. trachomatis (Kuo et al., 1972). A fluorescein-conjugated Chlamydia genus-specific monoclonal antibody was used for staining chlamydial inclusions. Two cell culture passages were carried out before isolation was considered negative. Serum antibody was assayed against the infecting serovar E organisms by the micro-immunofluorescence test (Wang et al., 1975). Fluorescein-conjugates of anti-human IgM or IgG (Hyland Laboratories, Los Angeles, CA, USA) were used in the indirect fluorescent antibody staining for serology.

Histological procedures. The Carnoy's fixed tissues were processed by conventional procedures, embedded in paraffin wax, sectioned and stained with haematoxylin and eosin. Verhoeff-van Gieson stain was used to stain tissues specifically for connective tissue elements. Before immunostaining for chlamydial inclusions, the tissue sections were deparaffinized and stained by indirect fluorescent antibody or avidin-biotin immunoperoxidase methods by using C. trachomatis-specific monoclonal antibody (Stephens et al., 1982). Monoclonal antibodies specific to human lymphocyte antigens that are known to cross-react with monkey antigens were used to identify the inflammatory infiltrate in fixed paraffin-wax embedded tissues. The antibodies included $\mathrm{L}_{26}$, a $\mathrm{B}$ lymphocyte marker (research antibody developed by Y. Ishii, T. Takami, H. Yuasa, T. Takei and K. Kikuchi, Sapporo Medical College, Sapporo, Japan), and T200, an anti-human pan lymphocyte marker against leucocyte common antigen (Dako M701, Santa Barbara, CA, USA). Fibroblasts were labelled by vimentin (43BE8 marker) (Dako), a marker of cells of mesothelial origin. The indirect immunoperoxidase staining method was used for all of these cells. For transmission electron microscopy, separate tissues were first fixed by Karnovsky's fixative and secondarily fixed by osmium tetroxide. Specimens were then stained with uranyl acetate, dehydrated through a graded series of alcohols, and embedded in Epon (Ernest F. Fullam, Schenectody, NY, USA) en bloc. Thin sections were stained with uranyl acetate and lead citrate, and viewed in a Philips 201 transmission electron microscope (Philips Electronic Instruments, Mahwah, NJ, USA).

\section{Results}

\section{Light microscopy}

Primary infection. At 2 days after the primary inoculation (Day 0), the submucosa (stroma) of the fimbrial tissues was moderately infiltrated with polymorphonuclear cells (PMNs) (Fig. 1a). At Days 3-4, lymphocytic cells, as well as PMNs and plasma cells had infiltrated the stroma and mucosa. The inflammation was widespread throughout the tissues, and the lymphocytic cells had already begun to migrate from the submucosa up into the mucosal epithelial layer. An exudate containing mixed inflammatory cells was present in the lumen by Day 4. By Days 5 (Fig. Ib) and 7, the deeper stromal tissues were heavily infiltrated with mononuclear cells, primarily lymphocytes. The lymphocytes that had migrated up into the epithelium were closely associated with epithelial cells that appeared damaged, as indicated by vacuolization. At this time, many plasma cells were seen in the submucosa. The luminal exudate contained red blood cells, lymphocytes, and desquamated epithelial cells. By Days 10 and 14, the mononuclear cellular response remained marked. Plasma cells were scattered throughout the stroma. Lymphocytes now appeared to be forming small clusters within stromal tissues just beneath the epithelium and also around the capillary (perivascular cuffing) within the connective tissues of the cyst capsule that contained the implant. By Days 21 and 28, the inflammation was markedly reduced. However, few lymphocytes and 
plasma cells remained in the tissues. No inflammatory changes were observed in the tissues of the control pockets inoculated with u.v.-inactivated organisms or HeLa cell material, indicating that the inflammatory reaction was due to active infection.
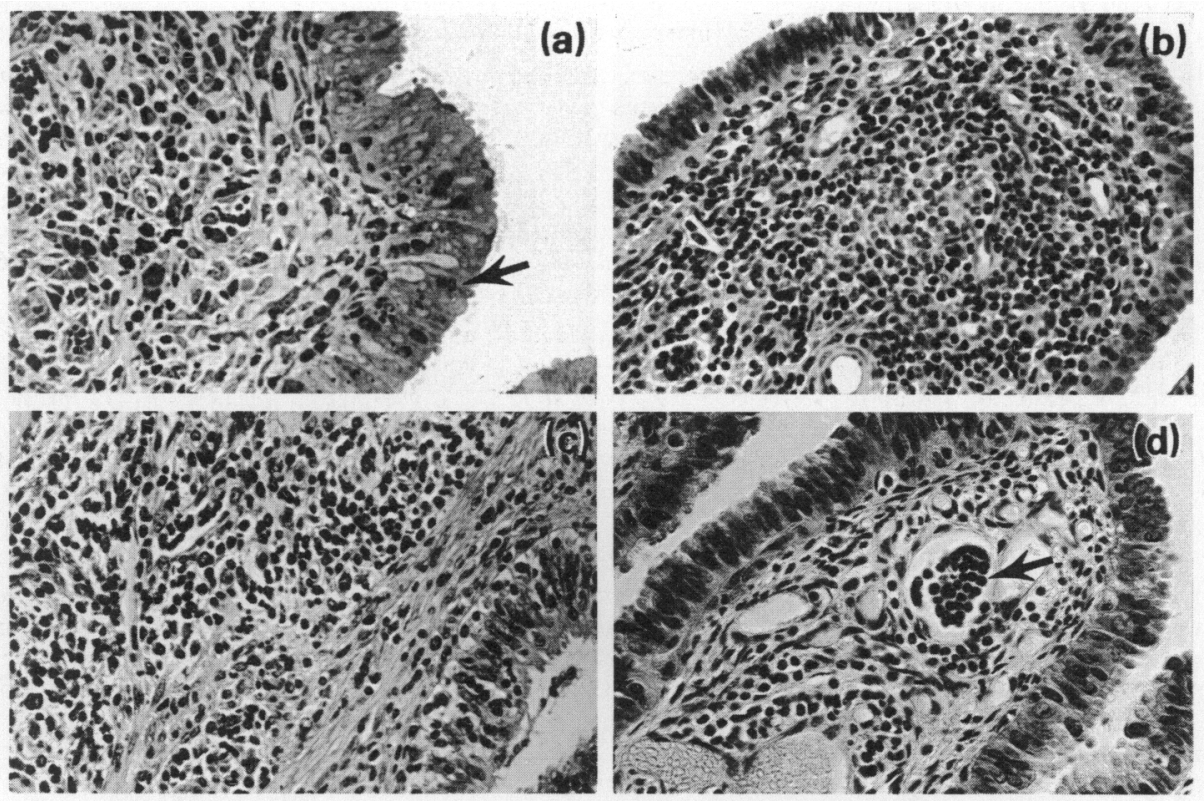

Fig. 1. Light microscopy of inflammatory responses in monkey fimbrial autotransplant pocket tissue after primary, secondary and tertiary chlamydial infection. (a) The inflammatory infiltrate is widespread in the submucosa. Capillaries contain numerous polymorphonuclear cells on Day 2. Note the ciliated and secretory epithelial cells of the mucosa and the polymorphonuclear infiltrate within the mucosa (arrows). $\times 40$. (b) The inflammatory infiltrate within the submucosa is predominantly mononuclear cells by Day 5 following a primary infection. The epithelium has thinned and contains numerous inflammatory cells. $\times 40$. (c) A dense widespread mononuclear cellular infiltrate characterizes the inflammatory response and permeates the submucosa by Day 2 after secondary infection. $\times 40$. (d) On Day 2 after tertiary infection in the submucosa, the infiltrate is predominated by mononuclear cells (primarily lymphocytes) and the capillaries are engorged and clogged with mononuclear cells (arrow). $\times 40$.

Secondary infection. A widespread mononuclear cellular infiltrate, with some areas of mixed mononuclear-PMN infiltration, was seen in the submucosa at Days 2 and 3 after the secondary rechallenge (Fig. 1c). Clustering of mononuclear cells in the submucosa was already apparent at Day 3. Perivascular cuffings with mononuclear cells were also evident in the outer stroma of the pockets. Exudate in the lumen contained mononuclear cells. Migration of mononuclear cells, with occasional PMNs, into the epithelium and destruction of epithelial cells were observed. At Days 5 and 7 , the mononuclear infiltration remained widespread, although it had begun to recede. The mononuclear cell infiltrate had become more localized to form lymphoid 'follicle-like' clusters. Numerous capillaries in the submucosa were clogged with mononuclear cells. Plasma cells increased in number in the submucosa. Epithelial cell damage appeared to subside even though mononuclear cells continued to migrate into the mucosal epithelium. At Day 10, mild mononuclear cell infiltration was present in the submucosa. Plasma cells were prominent. Numerous lymphoid follicle-like clusters were observed, especially in the connective tissue of the capsule. Some well established lymphoid follicles were noted (Figs $2 \mathrm{a}-\mathrm{d}$ ). The epithelial layers were relatively intact, 
but a few mononuclear cells continued to migrate into the epithelium. A mild to moderate degree of mononuclear cell infiltration still persisted in the subepithelium and deep stroma. Epithelial damage had generally been repaired although migration of mononuclear cells into the epithelium was still observed occasionally. Control tissues showed no inflammatory reaction.
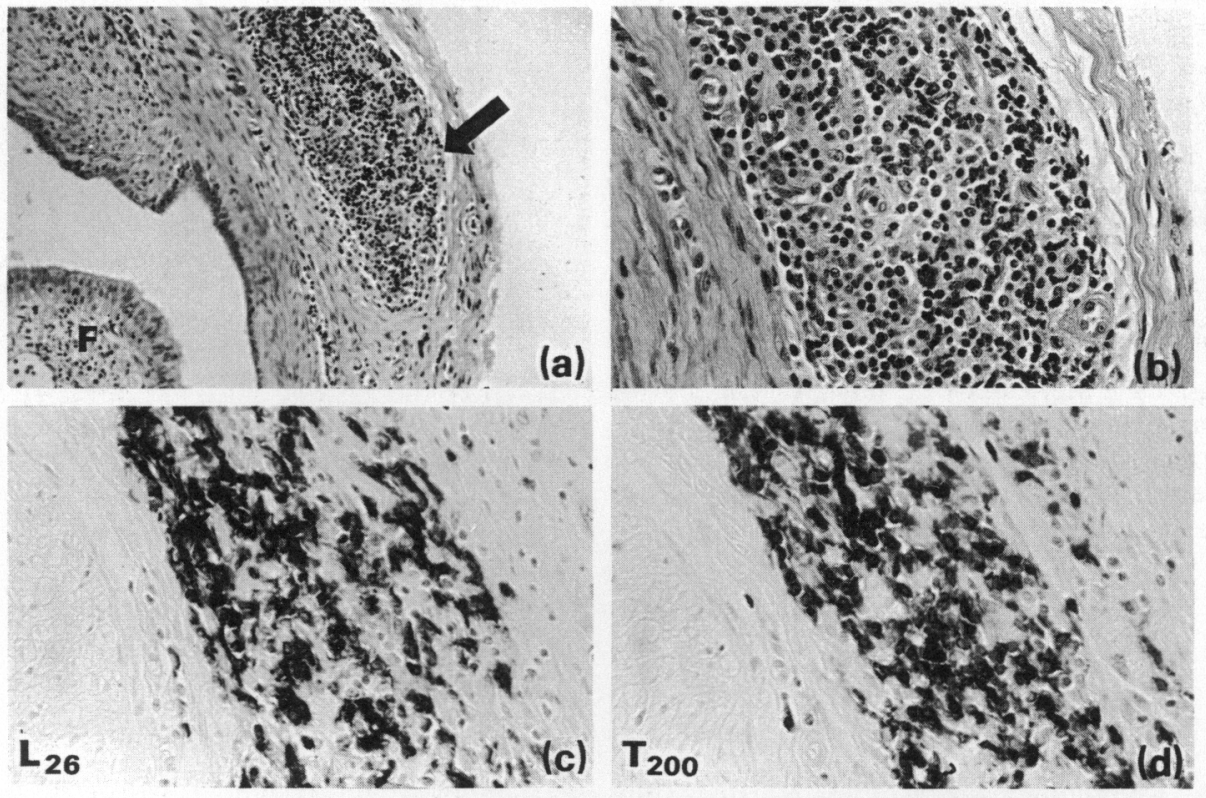

Fig. 2. Demonstration of lymphoid follicle formation in fimbrial autografts after secondary rechallenge with serovar $\mathrm{E}$ of $C$. trachomatis. The epithelium is intact and numerous lymphocytes are present in the submucosa. (a) A well defined lymphoid follicle (arrow) is shown in the deep stroma surrounding the pocket fimbria $(F)$ tissue. $\times 10$. (b) Higher magnification of the follicle. $\times 40$. (c) Immunoperoxidase staining revealed plasma cells within the follicle. The plasma cells are labelled by monoclonal antibody $\mathrm{L}_{26}$, a human $\mathrm{B}$ lymphocyte marker that cross-reacts with monkey tissue. (d) Immunoperoxidase staining also showed that $\mathrm{T}$ lymphocytes in the follicle were stained by the anti-human lymphocyte marker, $T_{200} \times 40$.

Tertiary infection. The inflammatory response was characteristically mononuclear in nature. At Day 2 after the third rechallenge, moderate numbers of mononuclear cells were observed in the subepithelial tissues and clogging of capillaries with mononuclear cells was prominent (Fig. 1d). Eosinophilia was also detected. Focal areas of epithelial destruction and luminal exudate containing mononuclear cells were noted. Extensive fibrosis was seen in the deep stromal tissue. At Day 5, severe mononuclear cell infiltration was observed in the subepithelium. Patchy areas of epithelial destruction were evident. Invasion of mononuclear cells into the epithelium continued and exudate containing mononuclear cells in the lumen persisted. Lymphoid follicles developed in the deep stroma. No other time intervals of rechallenge were studied.

\section{Immunocytochemical stains}

Antigen detection. Chlamydial inclusions were detected in secretory cells by both fluorescentantibody and immunoperoxidase stainings on Days 2, 3, 4, 5 and 7 after the primary inoculation. Chlamydiae were also present in the epithelium after secondary and tertiary challenges, although reisolation had become more difficult (Fig. 3a). 

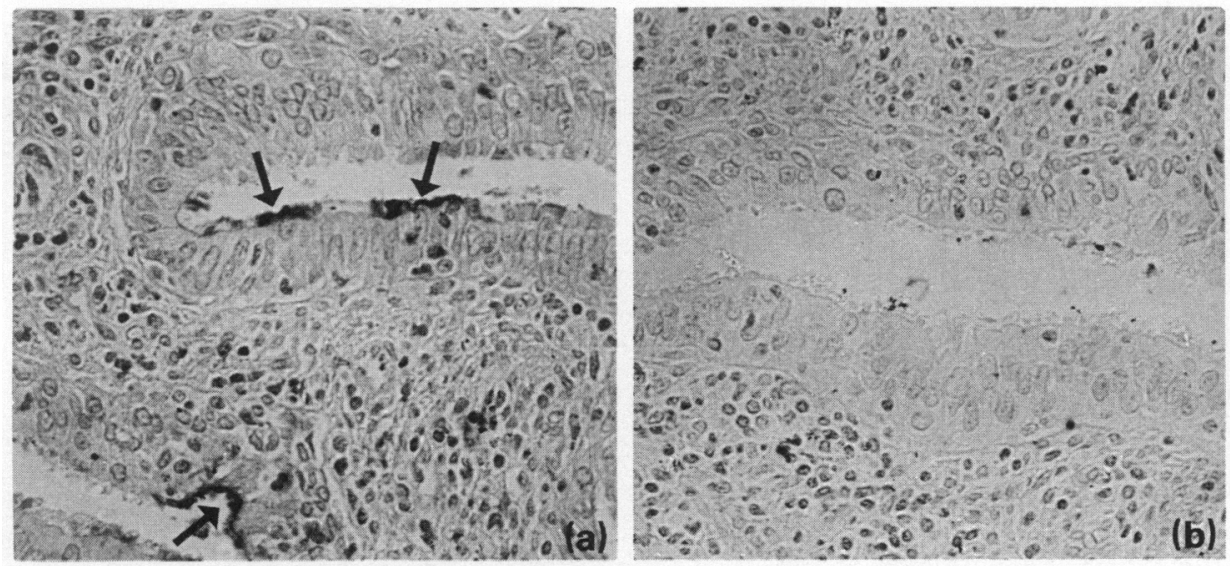

Fig. 3. Demonstration of chlamydial inclusions in fimbria epithelial cells. Tissues removed after Day 5 secondary infection were stained by immunoperoxidase using species-specific monoclonal antibody to $C$. trachomatis. (a) Many inclusions (arrows) are shown in the mucosa of the infected tissue. Note mononuclear cellular infiltrate in the submucosa tissue. $\times 40$. (b) Uninoculated tissues are negative. $\times 40$.
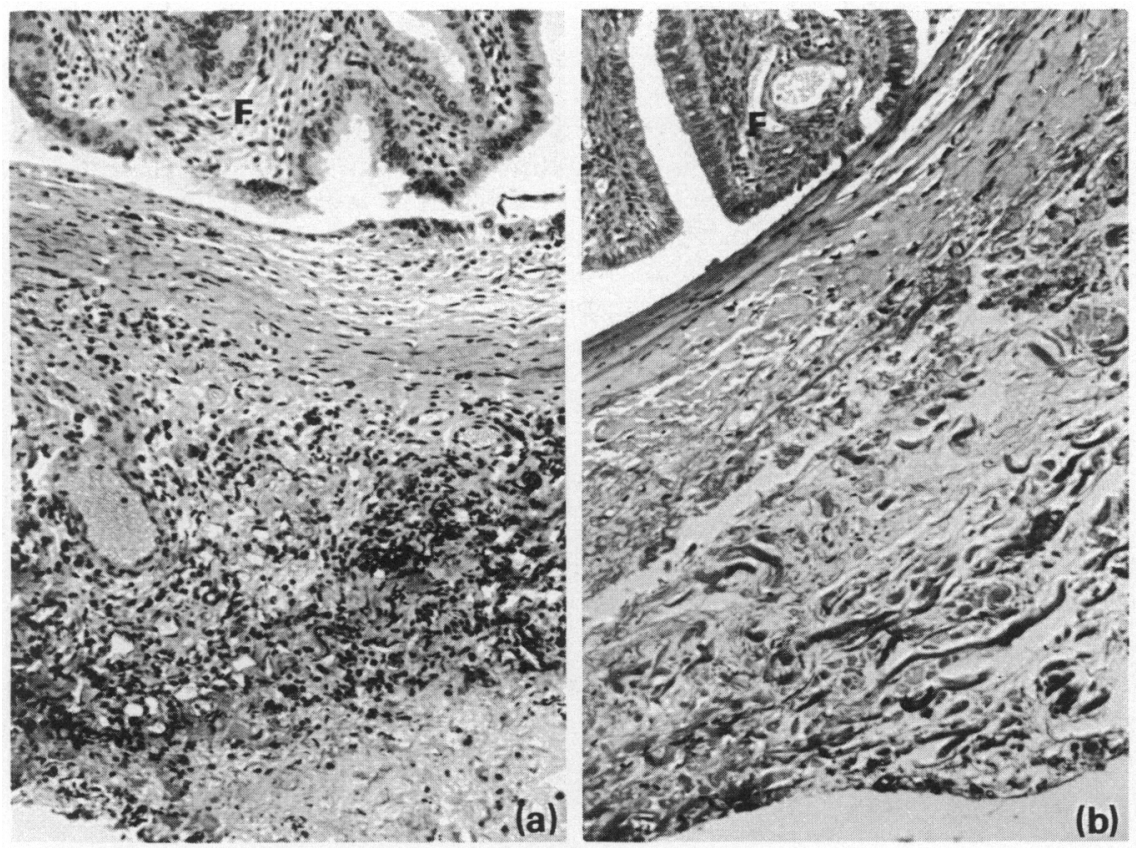

Fig. 4. Demonstration of fibrosis in fimbria pocket tissue following secondary chlamydial infection (a). Tissues were stained by Verhoeff-van Gieson stain to indicate an increase in elastin and collagen material after repeated infections. Note the contrast in deposits of black stain in the elastin and collagen as compared to the control. F: fimbria. $\times 16$. (b) The control tissue remains unresponsive. $\times 16$. 


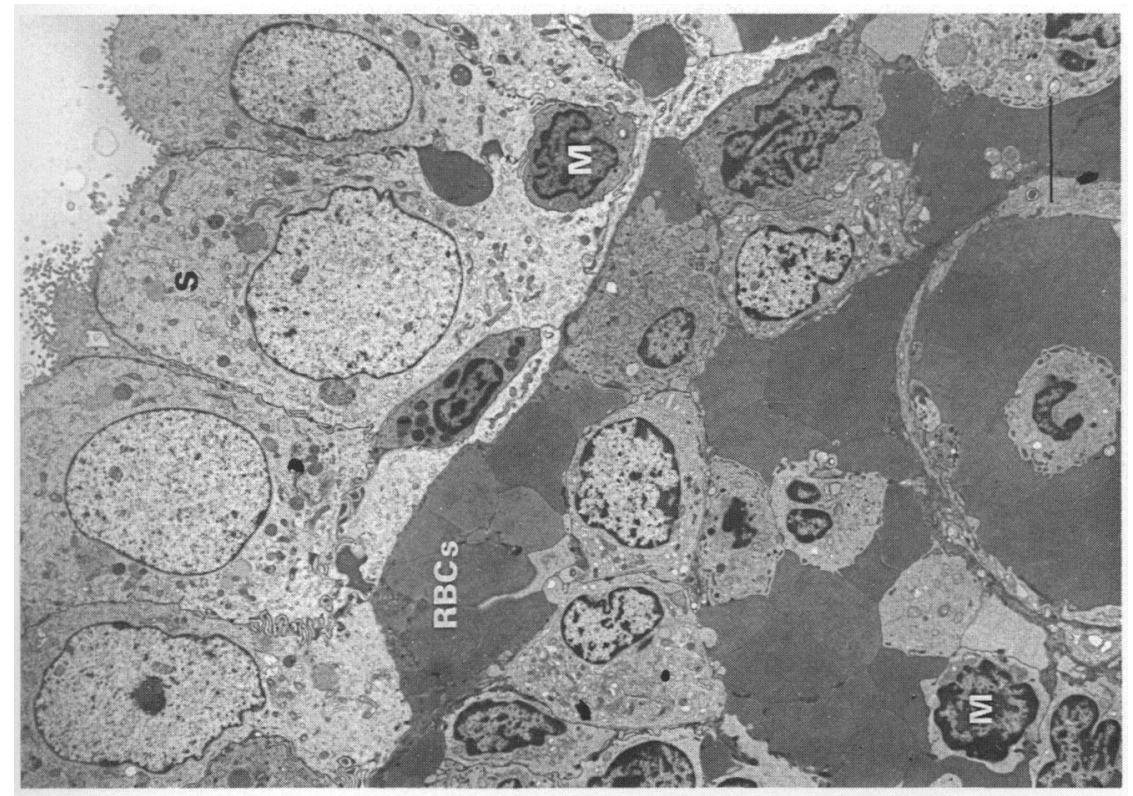

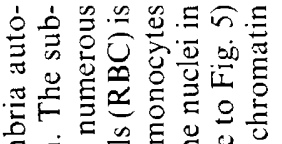

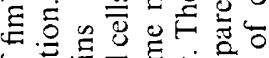

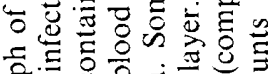

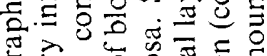

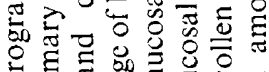

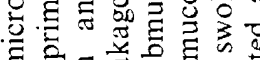

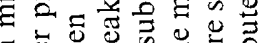

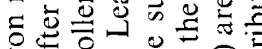

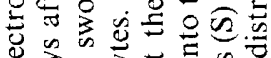

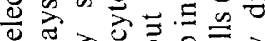

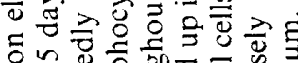

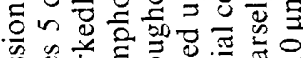

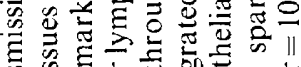

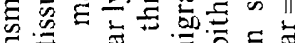

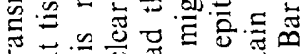

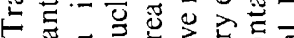

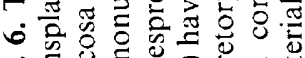

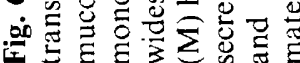

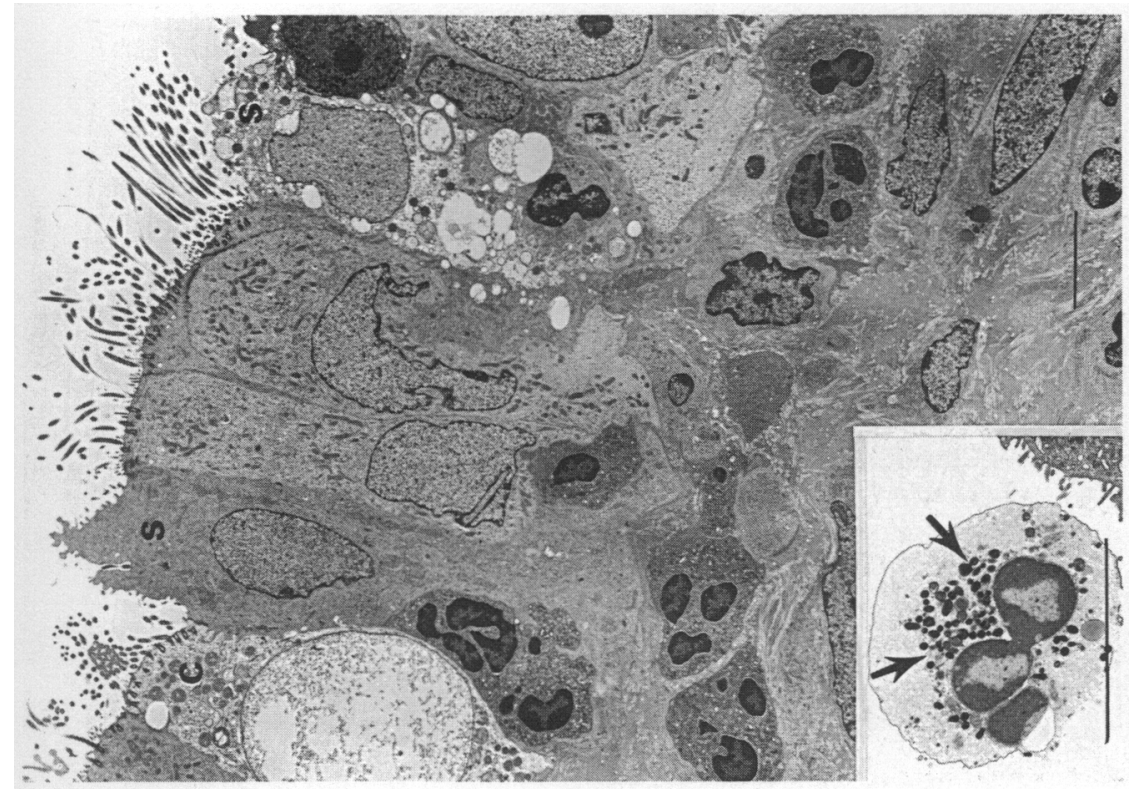

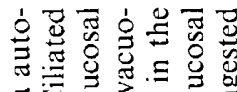
.

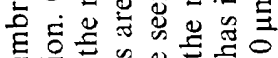
들

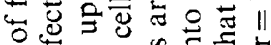

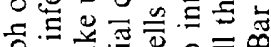

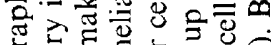
कo

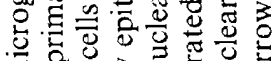

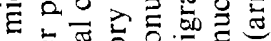

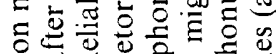
원

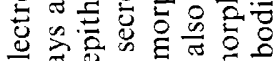

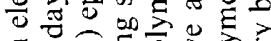

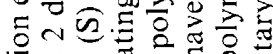

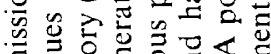

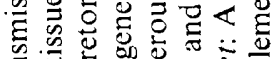

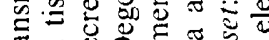

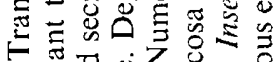
内

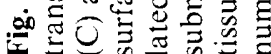




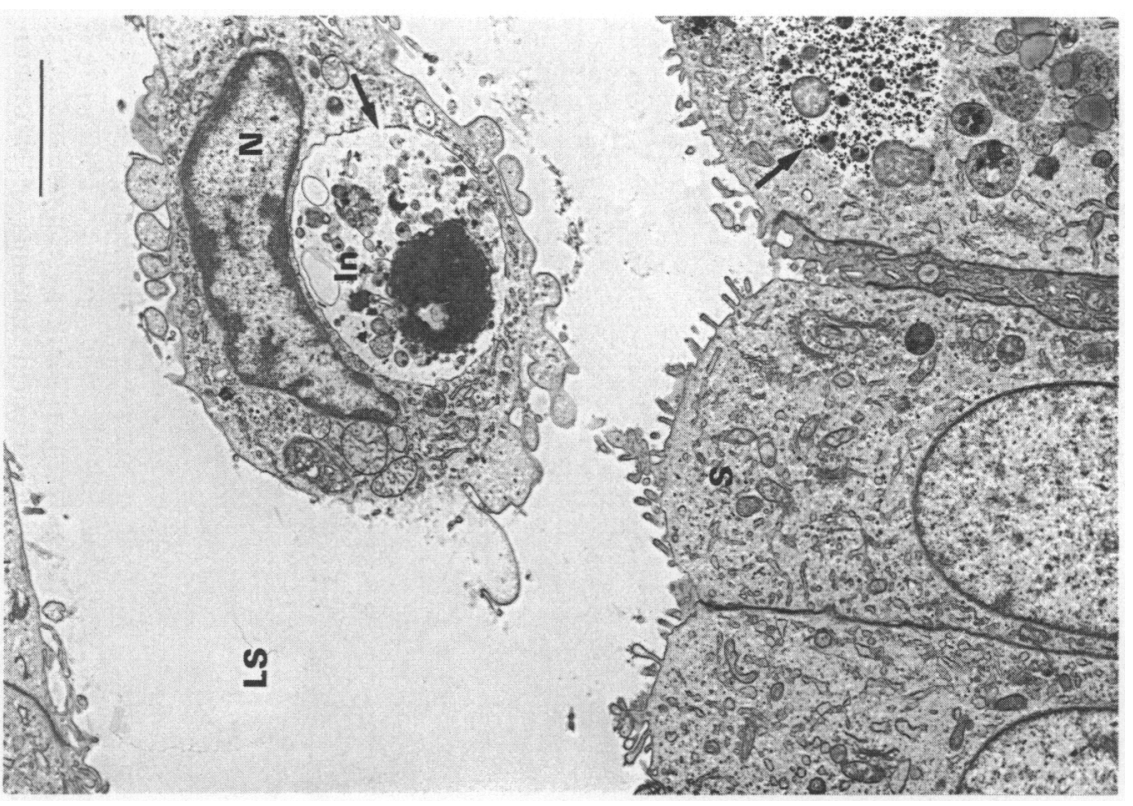

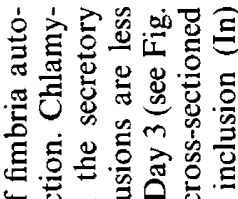

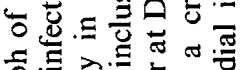
등.

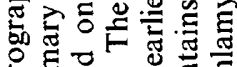

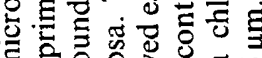
E $2000 \pi$ 的

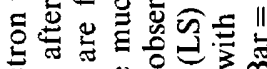

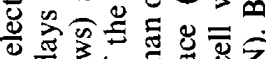
50

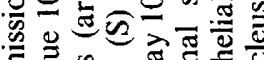

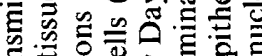

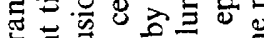

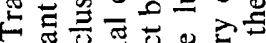

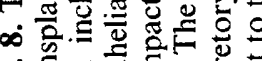

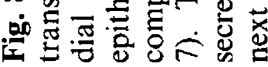

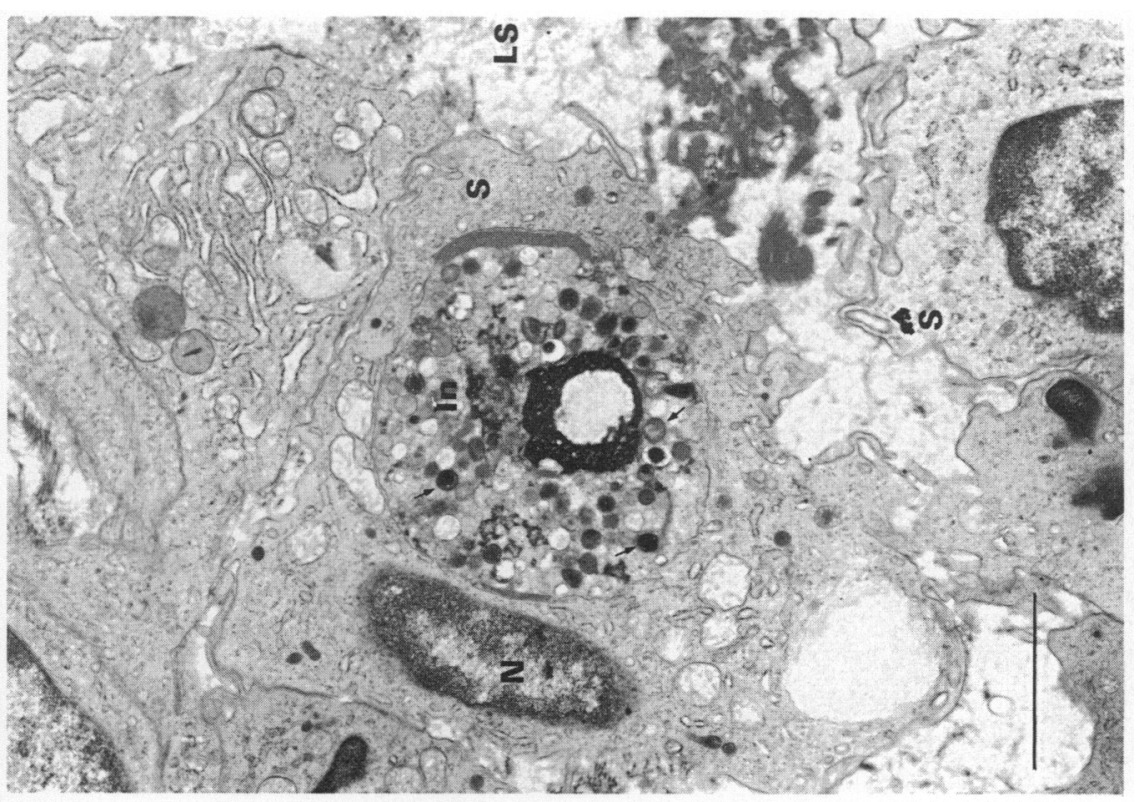

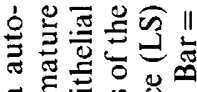
๘ $\varepsilon$ \& एँ

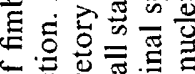
○远元西之 등.

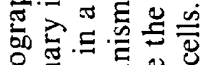

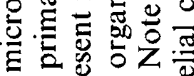

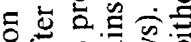

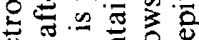

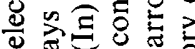

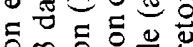
ธำ 흐원원

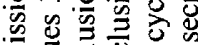

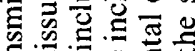
政运 可哥焉总

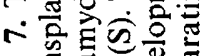

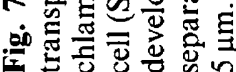


Immunocytology. Immunoperoxidase staining of $\mathrm{B}$ lymphocytes by $\mathrm{L}_{26}$ showed that the infiltrate in the submucosa contained increased numbers of plasma cells. Increases were more prominent following the secondary and tertiary infections. The staining revealed that mononuclear cells in the central portion of the lymphocyte clusters are mainly plasma cells (Fig. 2c). Staining with T200, a pan lymphocyte marker, showed that the mononuclear cell infiltrate also consisted of T-lymphocytes (Fig. 2d). T-cells were mainly seen in the cap portion of the lymphoid follicle. Fibroblasts were increased in number after the repeated infections as demonstrated by monoclonal antibody stain. Staining with Verhoeff-van Gieson stain indicated an increase in elastin and collagen materials (Figs 4a, b).

\section{Electron microscopy of primary infection}

Epithelial cell damage was observed early in the secretory and ciliated epithelial cells. Nuclear swelling and vacuolization of the intracellular organelles were present in both cell types. Degranulation and fragmentation of the endoplasmic reticulum had occurred in some of the cells (Fig. 5). Numerous PMNs were found close to the damaged cell at Days 2 and 3 after the primary inoculation and often contained partly digested elementary bodies (Fig. 5, inset). By Day 5, the infiltrating cells were predominantly mononuclear (Fig. 6). Capillaries within the submucosa were engorged with red blood cells. The RBCs had also migrated from the capillaries permeating the submucosa and mononuclear lymphocytes lined the endothelium (Fig. 6). Mononuclear lymphocytes had migrated up into the epithelial layer at this time. Lymphocytes, plasma cells and eosinophils had increased in number by Days 10 and 14. Mature chlamydial inclusions were identified in epithelial cells from fimbrial autografts by Day 3 (Fig. 7) and as long as Day 10 (Fig. 8) after infection. The inclusions observed at Day 10 were smaller and contained fewer elementary bodies. Inclusions were found in secretory but not in ciliated epithelial cells. In control tissues, the mucosa was healthy in appearance; the submucosa consisted of loose connective tissue without evidence of inflammation. The homogeneous cytoplasm contained numerous organelles and secretory granules. Tissues from reinfections were not available for electron microscopy studies.

\section{Microbiological and serological findings}

In the primary infection, C. trachomatis was reisolated from the fibrial transplants on Days 2, 3, 5 and 7 with the highest recovery rate on Day 2 (10 (83\%) of 12 pockets for 3 rhesus monkeys). The micro-immunofluorescence antibody response was demonstrated in these monkeys. Recovery of organisms from the pockets repeatedly inoculated was reduced. The recovery rates on Day 2 were 3 $(75 \%)$ of 4 ( 1 monkey) after secondary inoculation and 0 of 7 (2 monkeys) after the tertiary inoculation, indicating immunity. Cultures were not attempted for other time intervals for the reinfection experiments.

\section{Discussion}

We have described the histopathological findings of the inflammatory response during primary and repeated reinfections of salpingeal autografts with $C$. trachomatis in monkeys. The inflammatory response after primary inoculation was characterized by infiltration with PMNs in the acute phase and mononuclear cells in the chronic phase. However, the inflammatory response after repeated reinfections was dominated by a mononuclear cell infiltrate with follicle formation. These findings are similar to those described for primary infection (Ripa et al., 1979; Patton et al., 1983) and repeated reinfections (Patton et al., 1987b) in the intact monkeys in which C. trachomatis had been inoculated directly into the oviduct. However, we were able to monitor the temporally related 
events with the pocket model better than with the in-situ model. Using the pocket model, we showed that in primary infection the PMN leucocyte response, one of the earliest host responses against $C$. trachomatis, lasted for 2 days. The PMN infiltrate was quickly replaced with mononuclear cellular infiltrate by Day 5 with transition of a mixed PMN and mononuclear infiltrate between the two stages. The time of this event has not been clearly demonstrated in the in-situ model because serial sampling had not been conducted as early and frequently as desired (Ripa et al., 1979; Patton et al., 1983).

In contrast to the primary infection, the inflammatory response during repeated reinfections in the salpingeal autografts was predominantly mononuclear cellular infiltration throughout the course of infection and conspicuously lacked the initial PMN response. This finding underlines a strong cellular immune response following primary $C$. trachomatis infection. Other remarkable histopathological findings of repeated reinfections in the salpingeal autografts were plasma cell infiltration and lymphoid follicle formation. These findings are characteristically observed in ocular (Thygeson, 1934; Mitsui \& Suzuki, 1956; Dawson et al., 1962; Jin et al., 1980; Patton \& Taylor, 1986) and genital (Paavonen et al., 1982; Winkler et al., 1984; Patton et al., 1987b) infection with the trachoma serovar of $C$. trachomatis and have been described for natural infection in humans (Thygeson, 1934; Mitsui \& Suzuki, 1956; Jin et al., 1980; Paavonen et al., 1982; Winkler et al., 1984), as well as in experimental infections in monkeys (Dawson et al., 1962; Patton \& Taylor, 1986; Patton et al., 1987a, b). Plasma cells are typically found in large numbers in $C$. trachomatis conjunctivitis (Thygeson, 1934; Dawson et al., 1962), trachoma (Jin et al., 1980; Patton \& Taylor, 1986) and endometritis (Paavonen et al., 1982; Winkler et al., 1984). Follicle formation is also a prominent histopathological feature in C. trachomatis cervicitis (Paavonen, 1982), endometritis (Paavonen et al., 1982; Winkler et al., 1984), and salpingitis (Patton et al., 1987a, b).

Although the pocket model cannot reproduce tubal obstruction as in the in-situ model, we were able to use this model to study the immunopathogenesis of chronic salpingitis and changes that presumably lead to the tubal infertility. In the in-situ monkey model, a primary infection produces a self-limited acute salpingitis (Patton et al., 1983). However, chronic salpingitis with tubal scarring and distal obstruction is produced after repeated inoculation with $C$. trachomatis (Patton et al., 1987b). The histopathology and inflammatory responses in chronic salpingitis in these monkeys are characterized by extensive lymphoid cell infiltration, lymphoid follicle formation, and tissue destruction (Patton et al., 1987b). The same histopathological changes have been observed in repeated infections of the salpingeal autografts in this study. In addition, we used immunocytological and histochemical techniques to characterize inflammatory infiltrate and tissue damage and showed increased lymphocytic and plasma cell responses and increased fibroblast activity and extensive fibrosis, supporting our original hypothesis that after chlamydial infection the tissue damage is provoked by immune-mediated mechanisms (Grayston et al., 1985; Patton \& Taylor, 1986; Patton et al., 1987b).

Descriptions of histopathology of proven C. trachomatis salpingitis (Moller et al., 1979) and resulting tubal obstruction (Patton et al., 1984) are few. Human tissues are usually from women with established tubal infertility, i.e. occlusion without inflammation (Patton et al., 1984). Therefore, it is difficult to study the disease in humans. In this regard, the pocket model should be valuable for continued studies on the immunopathogenesis of $C$. trachomatis salpingitis, including scar formation. The effects of various factors such as antibiotics, hormones, and lymphokines can be evaluated in the pockets to determine whether prevention or reduction of the immunopathological events can preserve tubal fertility.

Reisolation results showed increasing difficulty in recovering organisms from inoculated pockets in repeated reinfections although organisms could be demonstrated in the epithelial tissues by immunological stains. Isolation of $C$. trachomatis from the conjunctiva in the chronic phase of trachoma (Kuo et al., 1977) and from the Fallopian tubes in women with chronic salpingitis and tubal infertility (Sellors et al., 1988) is similarly difficult. The intense cellular immune response provoked by reinfections may be a factor, suggesting a role for cellular immunity. 
We thank Patricia Cosgrove, Suzanne Allen, Yvonne Cosgrove and Peter Cummings for technical assistance.

This work was supported in part by research grants AI-22082 from the National Institute of Allergy and Infectious Diseases, and RR-00166 from the Division of Research Resources.

\section{References}

Dawson, C.R., Mordhorst C.H. \& Thygeson, P. (1962) Infection of rhesus and cynomolgus monkeys with egg-grown viruses of trachoma and inclusion conjunctivitis. Ann. N.Y. Acad. Sci. 98, 167-176.

Grayston, J.T., Wang, S.-P., Yeh, L.-J. \& Kuo, C.-C. (1985) Importance of reinfection in the pathogenesis of trachoma. Rev. Infect. Dis. 7, 717-725.

Jin, X., Zhang, X., Zhang, W., Wang, X., Sun, X., Zhao, W. \& Luo, S. (1980) Pathogenesis of trachoma. Chin. med. J. 93, 827-834.

Kuo, C.-C., Wang, S.-P., Wentworth, B.B. \& Grayston, J.T. (1972) Primary isolation of TRIC organisms in HeLa 229 cells treated with DEAE-dextran. J. Infect. Dis. 125, 665-668.

Kuo, C.-C., Wang, S.-P. \& Grayston, J.T. (1977) Growth of trachoma organisms in HeLa 229 cell culture. In Nongonococcal Urethritis and Related Infections, pp. 328-336. Eds D. Hobson \& K. K. Holmes. American Society for Microbiology, Washington, D.C.

Mitsui, Y. \& Suzuki, A. (1956) Electron microscopy of trachoma virus in section. Am. Med. Ass. Arch. Ophthalmol. 56, 429 448.

Moller, B.R., Westrom, L., Ahrons, S., Ripa, K.T., Svensson, L., von Mecklenburg, C., Henrikson H. \& Mardh, P.-A. (1979) Chlamydia trachomatis infection of the Fallopian tubes. Histological findings in two patients. Br. J. ven. Dis. 55, 422-428.

Paavonen, J., Vesterinen, E., Meyer, B. \& Saksela, E. (1982) Colposcopic and histologic findings in cervical chlamydial infection. Obstet. Gynecol. 59, 712-714.

Patton, D.L. \& Taylor, H.R. (1986) The histopathology of experimental trachoma: ultrastructural changes in the conjunctival epithelium. J. Infect. Dis. 153, 870-878.

Patton, D.L., Halbert, S.A., Kuo, C.-C., Wang, S.-P. \& Holmes, K.K. (1983) Host response to primary Chlamydia trachomatis infection of the fallopian tube in pig-tailed monkeys. Fert. Steril. 40, 825-840.
Patton, D.L., Moore, D.E., Spadoni, L.R. \& Soules, M.R. (1984) Active infection not detected at the time of tuboplasty. Fert. Steril. 41, 795, Abstr.

Patton, D.L., Kuo, C.-C., Wang, S.-P., Brenner, R.M., Sternfeld, M.D., Morse, S.A. \& Barnes, R.C. (1987a) Chlamydial infection of subcutaneous fimbrial transplants in cynomolgus and rhesus monkeys. J. Infect. Dis. 155, 229-235.

Patton, D.L., Kuo, C.-C., Wang, S.-P. \& Halbert, S.A. (1987b) Distal tubal obstruction induced by repeated Chlamydia trachomatis salpingeal infections in pigtailed macaques. J. Infect. Dis. 155, 1292-1299.

Ripa, K.T., Moller, B.R., Mardh, P.-A., Freundt, E.A. \& Melsen, F. (1979) Experimental acute salpingitis in grivet monkeys provoked by Chlamydia trachomatis. Acta path. microbiol. scand. Sect. B 87, 65-70.

Sellors, J.W., Mahony, J.B., Chernesky, M.A. \& Rath, D.J. (1988) Tubal factor infertility: an association with prior chlamydial infection and asymptomatic salpingitis. Fert. Steril. 49, 451-457.

Stephens, R.S., Kuo, C.-C.\& Tam, M.R. (1982) Sensitivity of immunofluorescence with monoclonal antibodies for detection of Chlamydia trachomatis inclusions in cell culture. J, clin. Microbiol. 16, 4-7.

Thygeson, P. (1934) The etiology of inclusion blenorrhea. Am. J. Ophthalmol. 17, 1019-1034

Wang, S.-P., Grayston, J.T., Alexander, E.R. \& Holmes, K.K. (1975) Simplified microimmunofluorescence test with trachoma-lymphogranuloma venereum (Chlamydia trachomatis) antigens for use as a screening test for antibody. J. clin. Microbiol. 1, 250-255.

Winkler, B., Gallo, L., Reumann, W., Richart, R.M., Mitao, M. \& Crum, C.P. (1984) Chlamydial endometritis: a histological and immunohistochemical analysis. Am. J. Surg. Pathol. 8, 771-778.

Received 1 August 1988 